INPLASY

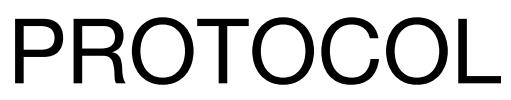

To cite: Zhou et al.

Comparative Efficacy and safety of Different Acupuncture Treatments for Functional Constipation: A network metaanalysis. Inplasy protocol 202070127. doi: 10.37766/inplasy2020.7.0127

Received: 29 July 2020

Published: 29 July 2020

Corresponding author: Ziquan Zhou

43096483@qq.com

Author Affiliation:

Acupuncture and Massage

College, Chengdu University of

Traditional Chinese Medicine.

Support: NFSC(NO.81774430).

Review Stage at time of this submission: The review has not yet started.

Conflicts of interest:

None of the authors have any conflicting interests.

\section{Comparative Efficacy and safety of Different Acupuncture Treatments for Functional Constipation: A network meta-analysis}

Zhou, Z1; Zou, Z2; You, X3; Wen, X'; Yao, J5; Li, Y6.

Review question / Objective: Nowadays, there are many randomized controlled trials and systematic reviews that show the acupuncture is effective for functional constipation(FC). As the diversity of acupuncture methods, there are lacking of relevant systematic evaluation literature to compare different acupuncture treatments and explain which is the best in efficacy and safety for FC.

Condition being studied: FC is a common clinical disease without any specific physiological that causes affects a considerable proportion of the population of all ages. The pathogenesis of $F C$ is very complicated, and its pathophysiological mechanism is not yet clear. FC can lead to abdominal pain, discomfort, gas, headache, nausea, and anorexia as well as potentially contributing to functional loss and length of stay in the hospital. In recent years, a large number of RCTs and meta-analyses have shown that acupuncture have certain advantages over conventional drugs in the treatment of functional constipation. However, due to the diversity of acupuncture methods, its relative effectiveness have not yet been studied and explained. Therefore, this study will compare the differences and rank in the efficacy of multi-acupuncture in the treatment of functional constipation, in order to provide a reference for clinical treatment.

INPLASY registration number: This protocol was registered with the International Platform of Registered Systematic Review and Meta-Analysis Protocols (INPLASY) on 29 July 2020 and was last updated on 29 July 2020 (registration number INPLASY202070127).

\section{INTRODUCTION}

Review question / Objective: Nowadays, there are many randomized controlled trials and systematic reviews that show the acupuncture is effective for functional constipation(FC). As the diversity of acupuncture methods, there are lacking of relevant systematic evaluation literature to compare different acupuncture treatments 
and explain which is the best in efficacy and safety for FC.

Condition being studied: FC is a common clinical disease without any specific physiological that causes affects a considerable proportion of the population of all ages.The pathogenesis of FC is very complicated, and its pathophysiological mechanism is not yet clear. FC can lead to abdominal pain, discomfort, gas, headache, nausea, and anorexia as well as potentially contributing to functional loss and length of stay in the hospital. In recent years, a large number of RCTs and metaanalyses have shown that acupuncture have certain advantages over conventional drugs in the treatment of functional constipation. However, due to the diversity of acupuncture methods, its relative effectiveness have not yet been studied and explained. Therefore, this study will compare the differences and rank in the efficacy of multi-acupuncture in the treatment of functional constipation, in order to provide a reference for clinical treatment.

\section{METHODS}

Participant or population: Studies involving patients with a confirmed clinical diagnosis of functional constipation (as diagnosed using any recognised diagnostic criteria, such as Rome II/ III / IV diagnostic criteria).If the Rome II/ III / IV diagnostic criteria was not mentioned, the participants will still be included when the study describes Rome II/ III / IV diagnostic criteria in detail.And it will be included regardless of age, gender, race, education status, nationality, economic status or severity and duration of disease, and excluded that participants have specific pathological causes(such as underlying structural or metabolic diseases) or pharmacologic causes(such as opioid related constipation or antipsychotics related constipation).

Intervention: Selection of acupuncture methods for analysis will include manual acupuncture(MA),electroacupuncture(EA), warm needling(WN), acupoint catgut e m b e d d i n g ( A C E), a c u p o i n t application (AA) alone, or a combination of any two of these methods or combinations of any of these acupuncture methods with conventional medications $(\mathrm{CM})$, regardless of acupoints selection or needling techniques.

Comparator: Any of included methods which is different from the invervention group will be included, as well as sham acupuncture, placebo, conventional medications(CM).

Study designs to be included: We will select randomized controlled trials published in English or Chinese without any regional restrictions.

Eligibility criteria: We will select randomized controlled trials published in English or Chinese without any regional restrictions. Literature reviews, animal studies, retrospective studies, case reports, academic dissertation, conference literature and studies with unavailable or incomplete data will be excluded.

Information sources: We will search the following electronic databases: PubMed, Web of Science, EMBASE, The Cochrane Library, China National Knowledge Infrastructure (CNKI), WANFANG database (Chinese Medicine Premier), and Chinese Biomedical Literature database (CBM). The search strategy will include only terms relating to or describing the intervention, and MeSH and free text terms will be used to identify relevant literature. The language of the studies published is limited to English or Chinese.Search dates: data from their inception to 30,June 2020.

Main outcome(s): The main outcome is Complete Spontaneous Bowel Movements(CSBMs) that refers to the times of defecation that can be completely eliminated without laxative or manipulation.And effect of measures is mean complete spontaneous bowel movements from baseline to the end of treatment. 
Additional outcome(s): The additional outcomes are The Bristol score and adverse events. The Bristol score which values of 1 or 2 indicate constipation, 3 and 4 are considered normal, and 5 to 7 are indicative of diarrhea or urgency and mean The Bristol score from baseline to the end of treatment as the effect of measures.

Quality assessment / Risk of bias analysis: The Risk of bias (quality) will be assessed by two researchers with reference to the Cochrane Collaboration Risk of Bias Tool 5.1.0. The following characteristics will be assessed: random sequence generation, allocation concealment, blinding of participants and personnel, blinding of outcome assessment, incomplete outcome data, selective reporting, other bias. In the process of evaluation, if there are disagreements, they will be resolved through discussion or a third reviewer.

Strategy of data synthesis: We will conduct a network meta-analysis to compare the effects of different acupuncture treatments for FC. The network meta-analysis combined direct and indirect evidence to assess the relative effectiveness of different acupuncture treatments, even if the interventions were never directly compared in a randomized controlled trial, and ranked the interventions. The analyses will be conducted by using Stata, WinBUGS and RevMan software.

Subgroup analysis: If necessary, the authors will carry out subgroup analysis. For example, we could conduct subgroup analysis based on the types or forms of acupuncture intervention, the treatment period, duration of disease etc.

Sensibility analysis: If there is a large heterogeneity, we will use the method of meta regression for sensitivity analysis, or we will exclude the studies one by one for sensitivity analysis in the direct comparison.

Language: Languages will be limited to Chinese and English.
Keywords: Functional Constipation; Acupuncture Treatments; Efficacy; Safety; network meta-analysis.

Contributions of each author:

Author 1 - Ziquan Zhou - Zhou Ziquan designed the study, and will review the results, interpret the data,draft the manuscript.

Author 2 - Zihao Zou - Zou Zihao designed the study, and will review the results and perform the statistical analyses.

Author 3 - Xi You - You Xi will be responsible for literature search, data extraction and evaluation of the bias.

Author 4 - Xin Wen - Wen Xin will be responsible for literature search, data extraction and evaluation of the bias.

Author 5 - Junpeng Yao - Yao Junpeng will provide statistical expertise and guide the extraction of research data and the evaluation of bias.

Author 6 - Ying Li - Li Ying will provide financial support, read and approve the final manuscript.

Country(ies) involved: China. 